\title{
Numerical Linear Algebra in Applications
}

\section{Daniele Bertaccini*}

Università di Roma Tor Vergata, Dipartimento di Matematica, 00133 Roma, Italy

The cross-fertilization between numerical linear algebra and reallife world has been very fruitful and continuosly incresing in number and strength in the last decades. In particular, applications have been making increasingly sophisticated use of linear algebra on both theoretical and algorithmic fronts. The interaction between them has been growing, leading to many new algorithms. My attention in these years was and is concentrated on the approximations of large and sparse linear systems mainly generated by accurate discretization of partial differential equations and in these years many advances have been established.

On one hand, the need for "effective" (in the sense that can solve the problem but also in a relatively small wall clock time with the prescribed accuracy) algorithms is every day increasing, and the answers are every day more built around the given problem hybridizing various techniques; see, e.g., our recent numerical methods for the large problems in the inner ear diseases [1,2]. On the other hand, the issues related to sparse matrix software that are of interest to application scientists and industrial users are often fairly different from those on which the academic community is focused. For example, for an application scientist or an industrial user, improving robustness may be far more important than finding a method that would gain speed. Memory usage is also an important consideration, but is seldom accounted for in academic research on sparse matrix solvers.

As a last example, linear systems solved in applications are almost always part of some nonlinear iteration (e.g., Newton-like) or optimization loop. It is important to consider the coupling between the linear and nonlinear parts, instead of focusing on the linear systems alone, see, e.g., Bertaccini and Calvetti [3]. These issues require a much more spread ground of research that must be, at the same time, accurate and rigorous, but also fast and easy to access for everybody in every place, minimizing the time to reach the online publication. Of course, these goals are conflicting because it is not easy to accurately analyze in a short time a research paper, find its weakness, if any, and guarantee wide access.

This is the case of researchers based in developing countries, small companies that cannot pay for the expensive fees of journal subscriptions and many others, in particular in the present difficult moment for all the national economies. A clever way to try to overcome all these issues at the same time is the OMICS' Applied \& Computational Mathematics Open Access policy (see http://www.omicsonline.org/OpenAccess.php for details) and the special features that the publisher provides (see http://www.omicsonline.org/special-features.php), that can help also people with visual disabilities, an issues that is rarely considered by publishers.

\section{References}

1. Moleti A, Paternoster N, Bertaccini D, Sisto R, Sanjust F (2009) Otoacoustic emissions in time-domain solutions of nonlinear non-local cochlear models. J Acoust Soc Am 126: 2425-2536.

2. Bertaccini D, Sisto R (2011) Fast numerical solution of nonlinear nonlocal cochlear models. J Comput Phys 230: 2575-2587.

3. Bertaccini D, Calvetti D (2007) Fast simulation of solid tumors thermal ablation treatments with a 3D reaction diffusion model. Comp Biol Med 37: 1173-1182.
*Corresponding author: Prof. Daniele Bertaccini, Università di Roma Tor Vergata, Dipartimento di Matematica, 00133 Roma, Italy, E-mail: bertaccini@mat.uniroma2.it

Received March 09, 2012; Accepted March 12, 2012; Published March 16, 2012

Citation: Bertaccini D (2012) Numerical Linear Algebra in Applications. J Applied Computat Mathemat 1:e101. doi:10.4172/2168-9679.1000e101

Copyright: (c) 2012 Bertaccini D. This is an open-access article distributed under the terms of the Creative Commons Attribution License, which permits unrestricted use, distribution, and reproduction in any medium, provided the original author and source are credited. 